\title{
Network Controller Procedures in SONATA, A Large Scale All-optical WDM Network
}

\author{
A. Bianco, E. Leonardi, M. Mellia, F. Neri \\ Dipartimento di Elettronica, Politecnico di Torino, Italy \\ \{bianco,leonardi,mellia,neri\} @polito.it
}

\begin{abstract}
This paper describes the network architecture of an optical WDM network named SONATA, which has been proposed and will be demonstrated in the context of the European Union ACTS Program. In this nation-wide all-optical network, end-terminals access a single passive routing node via PONs using a TDMA/WDMA access scheme based on slot reservations. The centralized network controller runs resource allocation algorithms in order to avoid conflicts among end-terminals. Since the complexity of optimal solutions to the resource allocation problem at the network controller is very large, we propose in this paper simple heuristic algorithms to solve the network control problem, and study their performance through simulation. Results of the design of the signaling protocol for SONATA are also reported.
\end{abstract}

\section{INTRODUCTION}

The project SONATA aims at avoiding the need for large and fast switching electronic nodes in a high-speed nation-wide network. To reach this goal, the network structure and the layer architecture within the network have been drastically simplified. The new proposal consists of a single-layer network platform for end-to-end optical connections, able to serve a very large number of terminals for both business and residential customers over a nation-wide geographical area.

Previous proposals of WDM optical networks can be roughly classified into two broad categories: single-hop systems, providing a fixed connectivity (broadcasting is often considered, as in the case of networks built

*This work was partially funded by the European Union ACTS Program within Project AC351 SONATA.

The original version of this chapter was revised: The copyright line was incorrect. This has been corrected. The Erratum to this chapter is available at DOI: 10.1007/978-0-387-35410-1_19 
around a passive star coupler,) among terminals, which run access protocols to share transmission resources (see, e.g., [1]), and wavelength routing systems, usually based onto general mesh topologies, in which optical cross-connects can be programmed to setup optical data pipes between terminal pairs (see, e.g., [2] and [3]). SONATA attempts to combine the best features of these two approaches: on the one hand, it keeps the architectural simplicity of single-hop systems, and terminals actively contribute to share the transmission resources. On the other hand, the possibility of dynamically modifying the end-to-end connectivity provided by the network is introduced to efficiently use network resources. Note also that the latter feature is one of the major strenghts of optical networks with respect to electronic networks.

In the SONATA network, a large number of end-terminals, which, depending on the network usage, can be street cabinets, IP routers, LAN switches, or workstations, are grouped in a Passive Optical Network (PON) infrastructure. The network topology is based on a centralized Passive Wavelength-Routing Node (PWRN) with $N$ input and $N$ output ports, which provides full connectivity among PONs via a single dedicated wavelength channel for each pair of PONs. Wavelength converters arrays are used at the PWRN in order to dynamically increase the channel capacity among pairs of PONs, as described below.

This multiple-access network is based on WDMA/TDMA protocols and exploits time and wavelength agility at terminals in a very simple network structure, where the single PWRN node provides passive routing functions and actively controlled wavelength conversion. The network control is centralized, and its primary goal is to assign time and wavelength resources to terminals: a signaling protocol that allows terminals to require both connection-oriented and connectionless services has been designed. The resource assignment performed at the Network Controller (NC) avoids any conflict among transmitters and receivers when transmitting data.

In SONATA, the physical switching function is removed from network nodes and distributed at terminals. Although a centralized network control is required, and active wavelength conversion is performed inside the network, we refer to the SONATA network structure as a "switch-less" network, in the sense that neither purely electronic switching nodes or cross-connects (telephony, IP, ATM, SDH), nor optical cross-connects (except for the wavelength routing node) are required within the network. Moreover, the network is completely buffer-less. This approach provides major network architecture simplifications and hardware reductions. 
We do not tackle issues related to the SONATA network feasibility in this paper: we do not discuss neither the components that should be used nor the physical limitations that should be taken into account when dimensioning the network. All these issues have been deeply analyzed in the SONATA project, and are discussed in $[4,5,6]$.

The paper focus is on algorithms that must be executed at the NC to solve the resource allocation problem. We first provide a more detailed description of the switch-less network architecture. Then, we discuss the time/wavelength resource allocation problem at the Network Controller. It can be shown that an Integer Linear Programming (ILP) formulation of the problem is possible, and that its optimal solution is in general NP-hard. We therefore propose a simple heuristic to solve the problem of resource allocation: we decouple the time dimension from the wavelength dimension, thus splitting the problem into two sub-problems: the scheduling of terminal requests in the time domain given a PON-to-PON channel assignment, and the design of the logical topology, i.e., the assignment of wavelengths to PONs via a proper setting of wavelength converters. The performance of the proposed algorithms is studied via simulation.

\section{NETWORK ARCHITECTURE}

A more detailed description of the network architecture is provided in this section. Recall that the SONATA switch-less network has the target of performing the concentration/distribution, switching and routing functions within a single network layer by providing end-to-end optical connections between a large number of terminals over a wide geographical area.

The structure of the switch-less network is depicted in Fig. 1. Terminals equipped with fast tunable transmitters and receivers are attached to Passive Optical Network (PON) infrastructures. Each PON is directly connected to an input and an output port of the single Passive Wavelength-Routing Node (PWRN). The PWRN has $N$ input and $N$ output ports, i.e., it is an $N \times N$ wavelength multiplexer. Logically, the behavior of the PWRN is such that wavelength $\lambda_{k}$ at input $i$ is routed to output $j=|i+k|_{N}$. Hence wavelength channels $0,1,2, \ldots, N-1$ on input port $i$ lead to output ports $i, i+1, i+2, \ldots, i-1$, and, on output fiber $j$, wavelength channels $0,1,2, \ldots, N-1$, carry information originated at input $j, j-1, j-2, \ldots, j+1$.

Each wavelength is shared between all terminals belonging to the same PON. A TDMA control protocol is therefore required. A global synchronization is available, and transmission is organized in WDMA/TDMA 


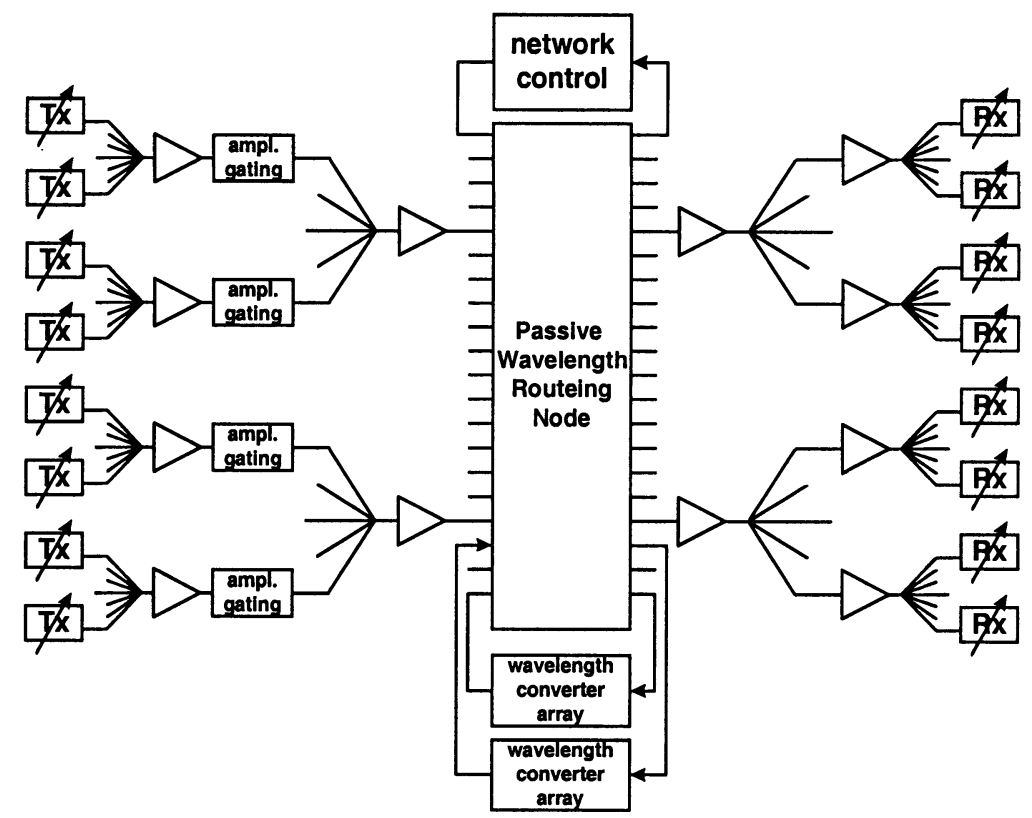

Figure 1 SONATA network architecture

frames. Each frame is composed of $F$ fixed-length slots. Any terminal wishing to communicate with another terminal simply tunes, in the allocated time slots, its transmitter and receiver to a known wavelength carrying multiplexed traffic through the PWRN between the pair of PONs to which the transmitting and receiving terminals are attached. Only one tunable transmitter/receiver pair per terminal (plus a fixed transmitter/receiver for signaling) therefore permits data communication between a given terminal and all other terminals in the network, regardless of the network size. Note that multicast transmissions to several terminals belonging to the same PON can be supported.

Although all connections can be made directly through the PWRN, as the traffic volumes grow towards the capacity limit of the network, a high degree of flexibility in allocating capacity can be obtained with actively controlled wavelength converter arrays. As shown in Fig. 1, these devices can be added, as required, around the PWRN, and connected to a certain number $\left(N_{d}\right)$ of its auxiliary ports (called "dummy ports"). On each PON, a wavelength channel is available to reach a particular dummy port, hence a wavelength converter array. From each array, one wavelength channel is provided to reach every output PON. Hence information from PON $i$ to PON $j$ can be routed on the direct channel 
$|j-i|_{N}$, and on up to $N_{d}$ additional channels going through wavelength conversion. In this way a variable number of wavelengths, depending on traffic requirements, can be dynamically allocated between a pair of PONs. Thus, the network structure depicted in Fig. 1 provides a full mesh topology between PONs through direct connections, and a large number of additional connections through wavelength converter arrays.

Additional $N_{c}$ ports of the PWRN interconnect network terminals with the centralized Network Control (NC) device, which is responsible for allocating time slots and wavelength channels to terminal requests.

The optimal fisical solutions for a "switch-less" network depend on the size of the network itself. Triangles in Fig. 1 represent optical wideband amplifiers. Amplifier gating blocks are used to eliminate (using SOA gates) noise from wavelength channels where no transmission occurs (see [4]). Global synchronization is obtained with a distribution of reference lines, and with the implementation of ranging functions to compensate for variable propagation delays between terminals and the PWRN.

As discussed in [4], a feasible set of characteristics for a large scale switch-less network, which can provide a communication infrastructure to terminals at the customer premises distributed nationwide, could be the following (assuming $N_{c}=1$ ):

- $N_{t}=20$ million terminals

- 1,000 $\mathrm{km}$ maximum distance between terminals

- 400 PONs with 50,000 terminals each

- up to $N_{d}=400$ wavelength converter arrays with 400 wavelength converter modules each

- $801 \times 801$ wavelength router with $\mathrm{W}=801$ wavelength channels on each fiber

- $10 \mathrm{Mbit} / \mathrm{s}$ total average rate per terminal

- $622 \mathrm{Mbit} / \mathrm{s}$ maximum rate per terminal

- $200 \mathrm{Tbit} / \mathrm{s}$ maximum network throughput

- $F=1,000$ slots per frame

- $10 \mu s$ of slot time, with $1 \mu s$ devoted to guard time

The drastic simplification of the network structure eliminates critical complex network components (note that the infrastructure is mainly passive), thereby increasing network reliability. No failure protection is 


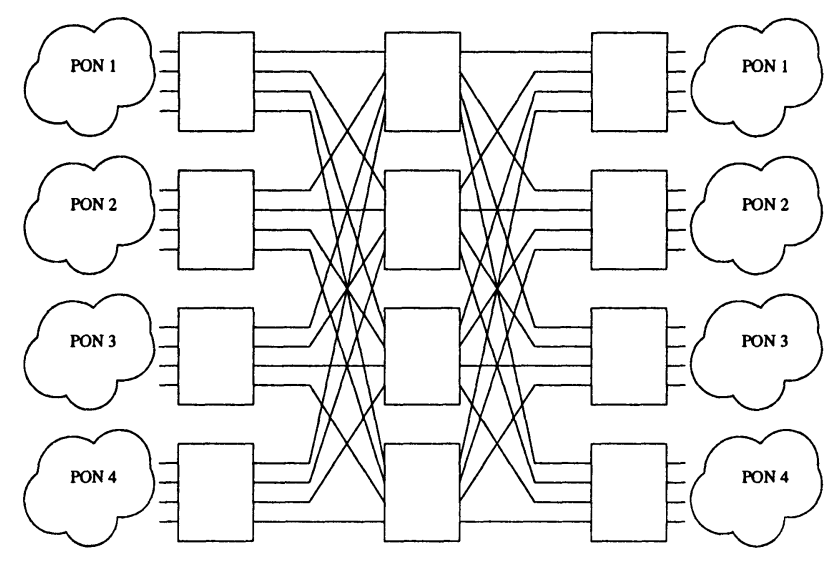

Figure 2 Three stage equivalent of the programmable portion of the SONATA network

however provided in the architecture of Fig. 1, and critical components (for example the Network Controller) may have to be duplicated for reliability in real implementations.

Logical Topology. The first $N_{p}$ ports of the PWRN provide a "wired" (fixed) full-mesh connectivity between PONs, while the additional $N_{d}$ dummy ports add a "programmable" connectivity between PONs. Fig. 2 shows the equivalent logical topology provided in the programmable portion of the PWRN in the case $N_{d}=N_{p}=4$. Terminals in PON $i$ can reach any of the $N_{d}$ wavelength converter arrays by tuning to the proper wavelength channel. This tuning capability corresponds to the first $\lambda$-switching stage in the logical topology. The individual wavelength converters can be configured in order to route input information to any output PON, leading to the second $\lambda$-switching stage in Fig. 2. The receivers in each PON can tune to any of the $N_{d}$ wavelengths routed to the PON by the wavelength converter arrays, leading to the third $\lambda$ switching stage. The resulting logical topology is a three-stage $\lambda-\lambda-\lambda$ switch, i.e., a Clos interconnection network. It is well known from the Slepian-Duguid theorem [7] that this three-stage interconnection structure is rearrangeable non-blocking when $N_{d} \geq N_{p}$, as it is normally the case for SONATA. Note that the interconnection between PONs is nonblocking, while the interconnection between terminals is blocking, due to the multiplexing of many terminals in each PON (at most 800 out of 50,000 terminals can receive/transmit in each slot) of a full-size network.

If we keep considering only the programmable portion of the network, the possibility of allocating transmission requests in different slots within 
the time frame can be viewed as a further switching stage in the time domain. Overall, the programmable portion of the switch-less network can be logically described as a five-stage $T-\lambda-\lambda-\lambda-T$ switch, which can be re-configured in every time slot.

Since time is divided into time slots, and each time slot can be used in a particular wavelength channel by only one of the terminals connected to the same PWRN port, a significant complexity is required in the NC operations. Considering a SONATA network deployed to its full capacity, the NC has to keep track of the state of $(N c+N d)^{2} \times F=$ $640,000,000$ time slots in every frame. Further constraints on the controller are due to the fact that the single transmitter/receiver pair has to be shared by all the connections which the terminal keeps active at the same time; more precisely, to permit a transmission from terminal $s$ to terminal $d$ in slot $k$, we must ensure that $s$ is not already transmitting in $k$, and also that $d$ is not already engaged in a reception in $k$. This requires in principle $2 \times N_{t} \times 1000=40,000,000,000$ state variables for each user.

\section{RESOURCE ALLOCATION ALGORITHMS IN SONATA}

The main task of the Network Controller is to allocate resources (time slots) to end-terminals, choosing a proper wavelength among the available ones.

Although globally optimal algorithms to allocate resources are possible, we propose in this paper a much simpler approach to resource allocation at the Network Controller, as the complexity of optimal control algorithms is so large that their implementation is impossible, given the very large network size. Moreover, the amount of signaling data that has to be sent to each terminal if optimal algorithms are used is very large, as mentioned in [5].

Our simple approach is based on the decoupling of the time dimension from the wavelength dimension. This means that we split the resource allocation problem into two sub-problems: scheduling of terminal requests in the time domain given a PON-to-PON channel assignment, and logical topology design of the network connectivity by properly assigning wavelengths to PONs via the wavelength converter arrays.

While scheduling is a task which must be done slot-by-slot, the logical topology design is assumed to be performed at a lower rate. Aiming at a sub-optimal approach with a limited complexity, we assume that the wavelength converters are reconfigured only once in a while (e.g., 
at frame boundaries) and that the scheduling algorithm operates on a given fixed logical topology.

We analyze in the sequel the two-steps of the resource allocation algorithm:

- Scheduling: first-fit allocation of slots given a fixed network connectivity;

- Logical Topology Design: reconfiguration of the network connectivity via wavelength converter arrays.

Scheduling. The scheduling algorithm is executed at the Network Controller on the basis of terminal requests received via signaling messages. Terminals can request slots according to two different modes:

- "persistent" request: an integer number of slots in each frame is assigned at connection setup; this number can be changed (either increased or decreased) during the connection duration

- "non-persistent" request: a (possibly large) amount of slots is assigned to a datagram communication, without time constraints in terms of number of involved frames

Obviously, the two modes refer mainly to CBR (or slowly varying VBR) connection-oriented services, and to datagram services, respectively.

When terminal $s$ belonging to PON $S$ sends a request to the NC in order to communicate with terminal $d$ belonging to $\mathrm{PON} D$, the sequence of steps executed at the $\mathrm{NC}$ is the following.

- If terminal $s$ requires the set up of a new connection, the NC looks for a candidate wavelength channel by scanning a linked list of wavelength channels assigned to the pair of PON $S \rightarrow D$. The first element of the linked list is always the "wired" channel among PONs $S \rightarrow D$, and the remainder of the list is ordered in increasing order of channel utilization. The new allocation is attempted on the first channel of the list that satisfies the condition that the number of free slots exceeds by a given percentage $b$ the number of requested slots.

- If $s$ is requesting new slots for an already set up connection, the NC selects the wavelength channel used for this connection.

- On the selected wavelength channel, the NC counts the available slots, excluding among free slots those in which either the source terminal or the destination terminal are busy (possibly for transmitting/receiving data associated to different connections). 
- If the amount of free slots resulting from the previous operation is less than the terminal request, the NC looks for another wavelength channel (only for a new connection request).

- If none of the wavelength channels already used for the communication from PON $S$ to PON $D$ is usable, the request is not accepted.

- If the number of free slots matches the terminal request, the NC selects the first available slots in the selected channel (first-fit allocation).

- In the case of datagram request, if the number of available free slots is less than the number of data slots required by the endterminal, the NC could also decide to operate a partial resources allocation: a threshold can be defined for this goal.

Fig. 3 provides a block diagram of the proposed scheduling algorithm.

For connection oriented (or persistent) requests, resources are allocated until one of the two involved terminals signals that it wants either to release some slots for this connection or to close it. For datagram (or non-persistent) requests, we assume that resources are allocated only in a single frame; in the next frame the NC will release all the resources previously allocated to datagram traffic.

Logical topology design. The logical topology design aims at obtaining an efficient allocation of available channels among PONs. The idea is quite simple: in order to reduce the slot allocation failure rate, the NC can "re-assign" channels: a channel showing a number of free slots above a given threshold $L$ can be freed, obviously re-routing connections previously assigned to that channel; free channels can be assigned to pairs of PONs experiencing a number of free slots below a given threshold $H$. We compute the load on each channel to decide when and whether or not to allocate/deallocate channels.

In every frame, a pair of PONs (a source PON $S$ and a destination PON $D$ ) is selected using a round-robin algorithm. We compute $L_{S D}$, the number of free slots on all the channels from PON $S$ to PON $D$. If $L_{S D}$ is larger than threshold $L$, we try to release a single channel, choosing the least loaded channel. If $L_{S D}$ is below threshold $H<L$, we try to assign another channel to the pair of PONs, provided that at least a free channel is available.

In the process of channel release, we try to release the least loaded channel. This implies a reallocation of all used slots in the selected channel. Only if the process is successful, i.e., all the slots can be allocated 


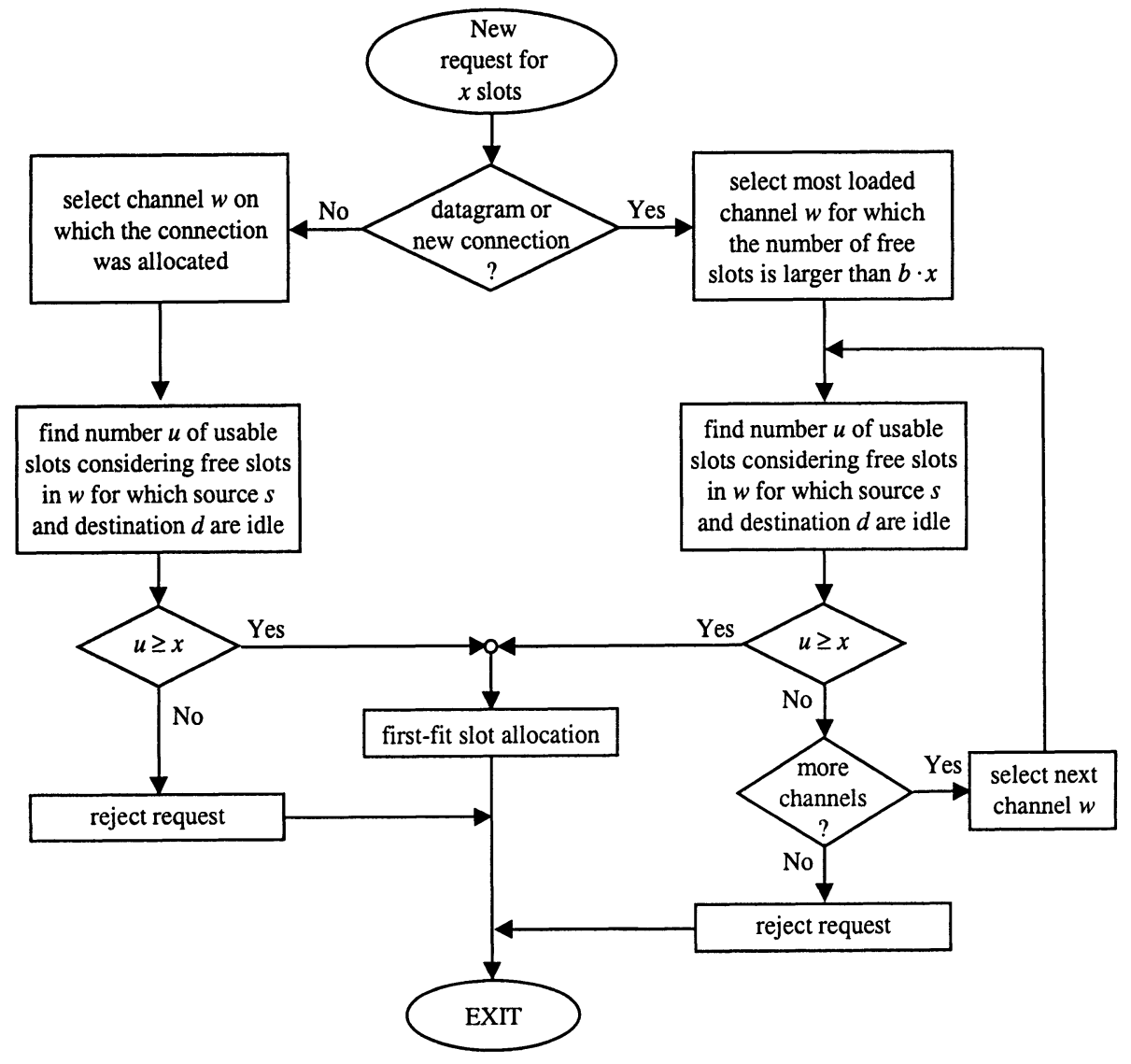

Figure 3 Block diagram for the scheduling algorithm

on other channels, the selected channel can be freed. We perform a complete search on all other channels allocated to the pair of PONs in order to reallocate every single slot, starting from the "wired" channel and continuing on all the other channels in the ordered list. If the reallocation process is successful, the channel can be freed and added to a list of available channels. Otherwise, if even a single slot is not successfully reassigned, the channel cannot be released. Note that, to be more precise, releasing a channel means releasing a wavelength on the transmitter side, and a wavelength on the receiver side.

When trying to add a channel we consider only a single channel addition between the PONs selected according to the round robin scheme. We first look for an available wavelength converter, i.e., a wavelength converter that is not used by both PON $S$ and PON $D$; if found, we 
choose the wavelength that should be used at the transmitter and at the receiver, and we logically add the channel to the pair of PONs.

If an available converter is not directly found, we check whether an available wavelength at both the transmitter and the receiver exists. If this is true, we know by Slepian-Duguid theorem [7] that we can rearrange the logical topology so that at the end of the process we obtain an available wavelength converter. Paull's algorithm [7] can be used to obtain the new logical topology, i.e., the new wavelength assignment. The latter algorithm is based on an iterative process that, after an upper bounded number of wavelength reassignments, guarantees the availability of a wavelength converter among the two PONs.

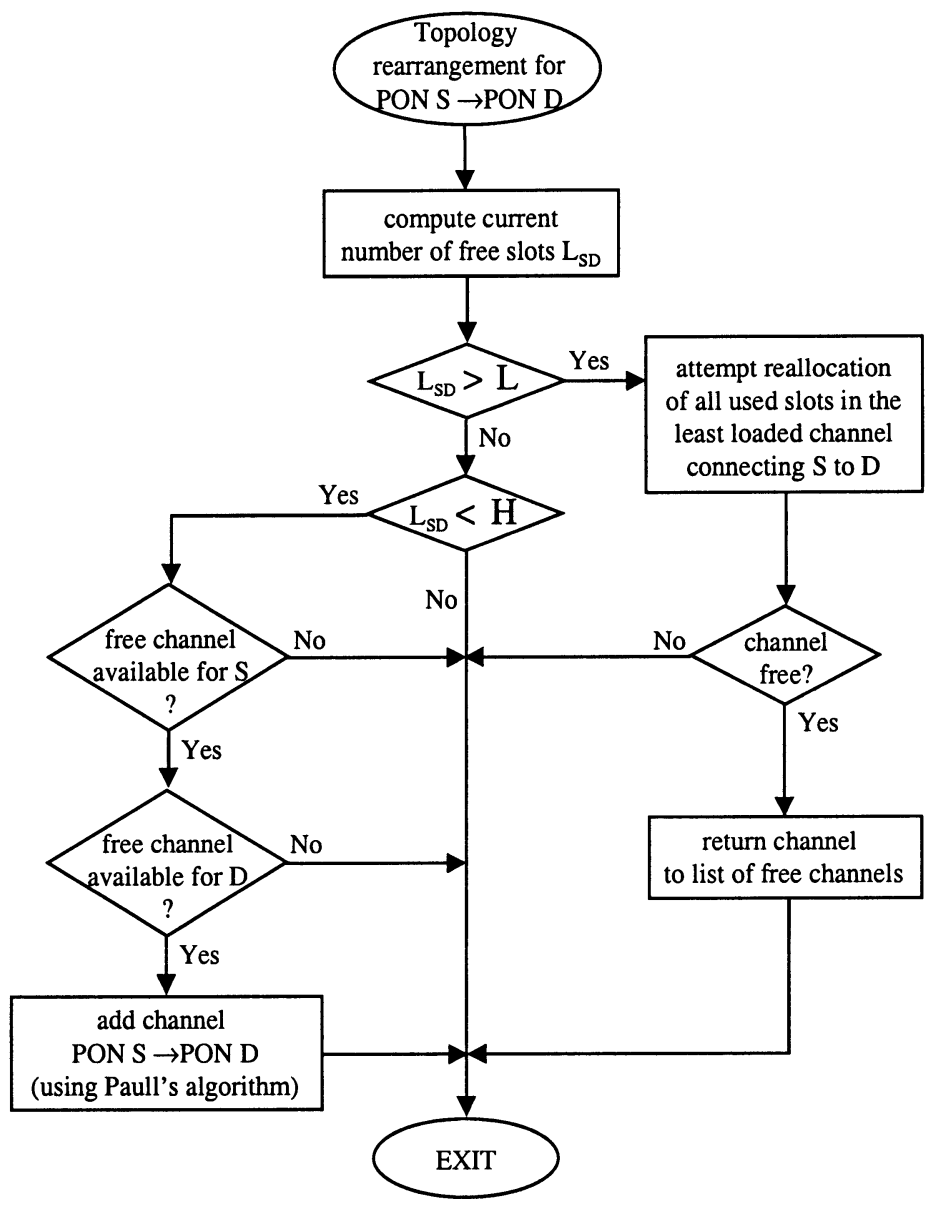

Figure 4 Block diagram for the logical topology design algorithm 
Fig. 4 provides a block diagram of the proposed logical topology design algorithm.

Note that each reassignment made while running the algorithm implies updating a significant amount of data structures, which are not reported here for the sake of conciseness. A more detailed description of the algorithms and of the data structures used at the Network Controller can be found in [6].

\section{SIGNALING PROTOCOL IN SONATA}

End-terminals in SONATA must reserve slots at the centralized Network Controller. Reservation is achieved through the exchange of signaling messages between end-terminals and the NC: $N_{c}$ (one or more) wavelength channels are available in each PON for signaling. Upstream signaling requires a medium access control strategy, due to the many contending terminals.

A detailed design of a signaling protocol for SONATA was performed in [5]. Signaling messages belong to the following classes:

- slot allocation request (upstream)

- slot de-allocation (upstream)

- slot allocation confirmation (downstream)

- slot de-allocation confirmation (downstream)

- slot allocation rearrangement, either in time or in wavelength, or both (downstream)

- wavelength converter configuration (downstream)

The format of these signaling messages is not reported here because of space limitations.

The use of connection identifiers to label different connection-oriented information flows at each end-terminal was proposed to ease control procedures, and to permit multiple connections between the same pair of end-terminals.

It has been shown that a fixed TDMA upstream access is a viable solution if slots can be partitioned in sub-slots on signaling channels. Indeed, by sub-slotting it is possible to obtain 50,000 minislots in each frame (using 50 sub-slots per slot). With $N_{c}=1$, up to 20,000,000 upstream signaling messages can reach the $\mathrm{NC}$ in one frame.

For what regards downstream signaling, with the proposed signaling messages, up to at most few thousand allocation requests can be confirmed in one slot. This means that, with $K_{c}=1$, up to a few million slot allocation requests can be confirmed in one frame. 
This evaluation of the bandwidth required for signaling is an important outcome of the design of the signaling protocol. Obviously, more bandwidth is required for downstream signaling than for upstream signaling. It is also easy to see that there is an almost mandatory need for a dedicated fixed receiver at each terminal for signaling (in addition to the tunable transceiver for data).

The optimal control of SONATA, in which wavelength converters can be reconfigured slot by slot, can be shown to require a large amount of signaling bandwidth (in addition to a very large complexity at the NC): approximately $1.5 \%$ of the bandwidth available for data transfers would have to be made available to downstream signaling in this case. Note that the proposed approach to network control assumes to let existing allocations unaffected by new allocations (this is often called "transparency" property in the technical literature), thereby significantly reducing the amount of downstream signaling.

The signaling bandwidth required for slot/topology rearrangements is instead negligible with respect to the bandwidth required for slot allocation/de-allocation (see $[5,6])$.

\section{SIMULATION OF THE PROPOSED RESOURCE ALLOCATION ALGORITHMS}

In this section we study by simulation the resource allocation algorithms, in terms of their speed of convergence to the optimal logical topology, their stability, and their capacity. By optimal topology we mean the topology that maximizes the network throughput in a given traffic scenario.

We first consider a small system, with 3 PONs, $N_{t}=150$ endterminals, i.e, 50 users per PON, frame length $F=100, N_{d}=3$ dummy ports connected to a bank of 3 wavelength converter arrays and 6 wavelengths per fiber. We modeled both network controller resource allocation algorithm, i.e., logical topology design and scheduling, under uniform traffic pattern. We discuss the speed of convergence of the algorithm to the optimal logical topology as a function of the system load. Note that in this case the optimal topology is obtained by allocating the same number (equal to 2) of channels to each PON pair.

At time 0 , all 9 additional channels provided by the programmable portion of the network are allocated between PONs connected to input and output ports with the same index, i.e., 4 channels are available between source PON $I$ and destination PON $I$ and 1 (wired) channel is available between PON $I$ and all other PONs. Thus, the topological distance is 12 , since each PON has 2 additional channels allocated to 
reach its end-terminals, and one channel missing towards the other two paths. Note that this is a logical topology that maximizes the topological distance $^{1}$ from the optimal topology under uniform traffic.

We show in Fig. 5 the topological distance of the current topology from the optimal topology as a function of time (one unit refer to a time frame), for variable loads. For medium-high loads $(\lambda=0.9)$, the system converges rapidly to the optimal topology; once the optimal topology is reached, due to traffic fluctuations, the algorithm sometimes modifies the logical topology, mainly releasing free channels; the topological distance from the optimum remains small. Results for smaller loads not reported for the sake of conciseness show a very similar behavior.

For moderate overload $(\lambda=1.4)$ the optimal topology is reached quite quickly; once all the channels are correctly allocated to pair of PONs, the system does not modify the topology since no channels become available at any time.

For sustained overload $(\lambda=2.4)$ the optimal topology is never reached; similarly to the previous scenario, once all the channels are allocated to pair of PONs, the system is not able to modify this topology since no channel becomes available at any time. However, the system does not converge in general to the optimal topology but to a topology that depends on the initial stochastic behavior of call requests.

To analyze the capacity provided by the proposed algorithms, we modeled again a 3 PON network, but with 10 users per PON and 10 slots per frame, subject to telephone traffic. Each call requires exactly one slot. A Poisson process models call arrivals, and call holding times are exponentially distributed with average 18.000 frames. Part of the traffic is uniformly distributed among PONs; the remaining traffic is randomly sent to terminals belonging to the PON to which the originating terminal belongs. The percentage of local traffic is taken as a parameter. The offered load is normalized with respect to the total number of slots available per frame, and the plotted throughput is the fraction of calls that are successfully routed through the SONATA network.

Figure 6 plots the network throughput under different locality factors; as a comparison, we added also a curve relative to the optimal scheduler that is able to route all calls up to the maximum number of available

${ }^{1}$ We define as topological distance between two topologies the following quantity:

$$
\sum_{S} \sum_{D}\left|C_{S D}^{1}-C_{S D}^{2}\right|
$$

where indexes $S$ and $D$ span over PONs, $C_{S D}^{1}$ and $C_{S D}^{2}$ represent the number of channels allocated from PON $S$ to PON $D$ in the first and in the second logical topology respectively. 


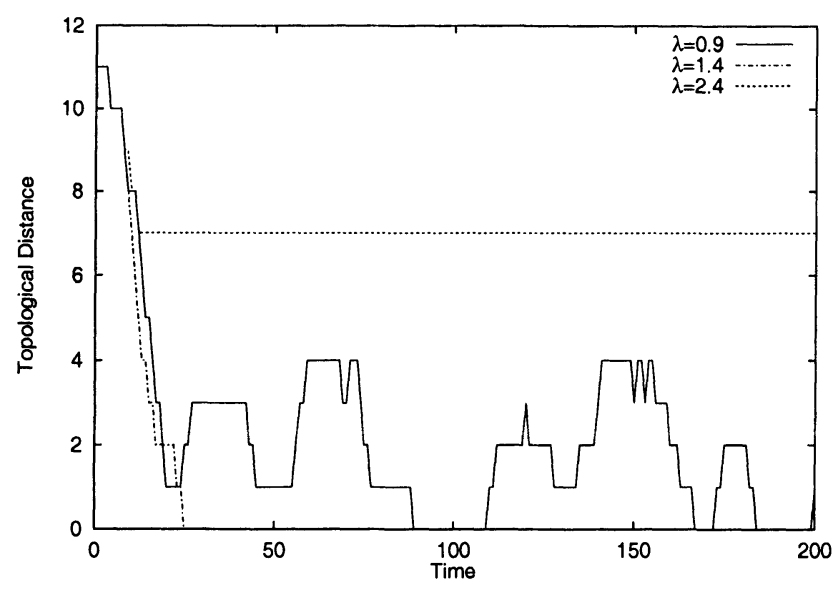

Figure 5 Convergence of the current logical topology to the reference topology

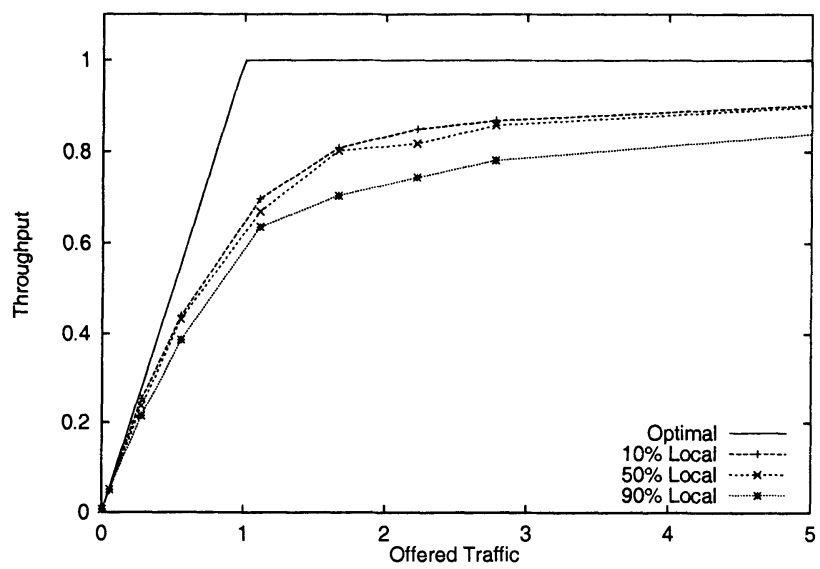

Figure 6 Throughput versus the offered load, with different traffic pattern.

slots, possibly completely reconfiguring the network at each frame, regardless of transmitter and receiver contentions. The performance of the proposed algorithms is not too far from the optimal behavior. When the amount of traffic locality is larger, a lower utilization of direct channels (which cannot be re-allocated according to traffic needs), hence a lower throughput, is experienced, as expected.

These results show that the network control algorithms proposed by the SONATA project lead to acceptable exploitation of the very large available bandwidth with a reasonable complexity. 


\section{CONCLUSIONS}

The paper described the network architecture and provided a performance analysis of a passive optical network named SONATA, which has been proposed and demonstrated in the context of the European Union ACTS Program. SONATA aims at avoiding the need for large and fast switching electronic nodes in a high-speed nation-wide network. To reach this goal, the network structure and the layer architecture within the network have been drastically simplified: the transport infrastructure consists of a single-layer of end-to-end optical connections. End-terminals fully exploit time and wavelength agility to exchange packetized information in the multiple-access network.

The control of the network is centralized at a network control device, whose primary goal is to assign time/wavelength resources to terminals in a way such that conflicts among transmitters and receivers are avoided. The paper focussed on the algorithms that must be executed at the Network Controller to solve the resource allocation problem.

Since the resource allocation problem at the Network Controller is in general NP-hard, we proposed in this paper simple heuristic algorithms that divide the solution into scheduling and logical topology design subproblems, aiming at a sub-optimal approach with a limited complexity.

An initial performance evaluation of the considered algorithms, based on simulation, was presented for a small scale system. 


\section{References}

[1] B. Mukherjee, "WDM-Based Local Lightwave Networks Part I: Single-Hop Systems", IEEE Network, vol.6, pp.12-27, May 1992.

[2] D. Banerjee, B. Mukherjee, "A Practical Approach for Routing and Wavelength Assignment in Large Wavelength-Routed Optical Networks", IEEE Journal on Selected Areas in Communications, vol. 14, n. 5, pp. 903-908, June 1996.

[3] R. Ramaswami, K. N. Sivarajan, "Design of Logical Topologies for Wavelength Routed Optical Networks," IEEE Journal on Selected Areas in Communications, vol. 14, n. 5, pp. 840-851, June 1996.

[4] M. Hill, N. P. Caponio, F. Neri, R. Sabella, "Single Layer Optical Platform Based on WDM/TDM Multiple Access for Large Scale "Switch-less" Networks", to be published in European Transactions on Telecommunications, Special Issue on WDM Networks, January 2000.

[5] A. Bianco, E. Leonardi, M. Mellia, M. Motisi, F. Neri, Specification of Signaling Functions in SONATA, ACTS Project SONATA AC-351, Working Document, Politecnico di Torino, 1999.

[6] A. Bianco, E. Leonardi, M. Motisi, F. Neri, Specification of Slot Allocation and Topology Design Protocols, ACTS Project SONATA AC351, Working Document, Politecnico di Torino, 1999.

[7] J. Hui, Switching and Traffic Theory for Integrated Broadband Networks, Kluwer Academic Publisher, 1990. 